\title{
Bleeding complications associated with the molecular adsorbent recirculating system: a retrospective study
}

\author{
Seon Woo Yoo ${ }^{1,2}$, Min-Jong Ki ${ }^{1,2}$, Dal Kim', Seul Ki Kim ${ }^{2,3}$, SeungYong Park ${ }^{2,3,4}$, Hyo Jin Han ${ }^{2,4}$, Heung Bum Lee ${ }^{2,3,4}$ \\ ${ }^{1}$ Department of Anesthesiology and Pain Medicine, Jeonbuk National University Hospital, Jeonbuk National University Medical School, Jeonju; ${ }^{2}$ Research Institute \\ of Clinical Medicine of Jeonbuk National University-Biomedical Research Institute of Jeonbuk National University Hospital, Jeonju; ${ }^{3}$ Humidifier Disinfectant \\ Health Center, Jeonbuk National University Hospital, Jeonbuk National University Medical School, Jeonju; ${ }^{4}$ Division of Respiratory and Critical Care Medicine, \\ Department of Internal Medicine, Research Center for Pulmonary Disorders, Jeonbuk National University Hospital, Jeonbuk National University Medical School, \\ Jeonju, Korea
}

Background: The molecular adsorbent recirculating system (MARS) is a hepatic replacement system that supports excretory liver function in patients with liver failure. However, since MARS has been employed in our hospital, bleeding complications have occurred in many patients during or after MARS. The objective of this study was to determine how MARS affects coagulopathy and identify specific factors associated with bleeding complications.

Methods: We retrospectively analyzed data from 17 patients undergoing a total of 41 MARS sessions. Complete blood count, coagulation profiles, and blood chemistry values were compared before and after MARS. To identify pre-MARS factors associated with increased bleeding after MARS, we divided patients into bleeder and non-bleeder groups and compared their pre-MARS laboratory values.

Results: MARS significantly reduced bilirubin and creatinine levels. MARS also increased prothrombin time and reduced platelet and fibrinogen, thus negatively impacting coagulation. PreMARS hemoglobin was significantly lower in the bleeder group than in the non-bleeder group $(P=0.015)$. When comparing the upper and lower 33\% of MARS sessions based on the hemoglobin reduction rate, hemoglobin reduction was significantly greater in MARS sessions involving patients with low pre-MARS international normalized ratio of prothrombin time (PT-INR) and factor $V$ $(\mathrm{P}=0.038$ and $\mathrm{P}=0.023$, respectively).

Conclusions: MARS could appears to alter coagulation-related factors such as factor $V$ and increase the risk of bleeding complications particularly in patient with low hemoglobin. However, individual differences among patients were large, and various factors, such as low hemoglobin, PTINR, and factor V levels, appear to be involved.

Key Words: artificial liver; blood coagulation disorders; end stage liver disease; extracorporeal life support; hemorrhage

\section{INTRODUCTION}

The treatment of liver failure encompasses symptomatic supportive care as a medical treatment and liver transplantation (LT) as a surgical treatment. The need for intensive critical care is based on the severity of symptoms, such as hemodynamic instability, encephalopathy,

\section{Original Article}

Received: March 5, 2021

Revised: July 2, 2021

Accepted: August 25, 2021

\section{Corresponding author}

Heung Bum Lee

Division of Respiratory and Critical

Care Medicine, Department of Internal Medicine, Research Center for Pulmonary Disorders, Jeonbuk National University Hospital, Jeonbuk National University Medical School, 20 Geonji-ro, Deokjin-gu, Jeonju, 54907, Korea

Tel: +82-63-250-1685

Fax: +82-63-254-1609

E-mail:Ihbmd@jbnu.ac.kr

Copyright (C) 2021 The Korean Society of Critical Care Medicine

This is an Open Access article distributed under the terms of Creative Attributions Non-Commercial License (https:// creativecommons.org/li-censes/by-nc/4.0/) which permits unrestricted noncommercial use, distribution, and reproduction in any medium, provided the original work is properly cited. 
bleeding, and hepatorenal syndrome [1]. LT should be considered upon progression to decompensated liver failure [2]. However, as liver failure progresses rapidly and exhibits a varying course, it is difficult to make early decisions regarding LT in practice. In addition, finding living donors for LT is difficult, and obtaining appropriate cadaveric donors takes a considerable amount of time.

The molecular adsorbent recirculating system (MARS) is a hepatic replacement system that supports excretory liver function in patients with liver failure. It is mainly used as a bridge therapy for patients waiting for LT $[3,4]$. MARS appears to improve bilirubinemia, hepatic encephalopathy, renal function, and systemic hemodynamics [5]. Although it may be a good option for patients with end-stage liver failure [6], many clinicians are reluctant to apply MARS due to its high price, various side effects, and lack of evidence that it reduces mortality and improves long-term survival rate [7-9]. Adverse effects of MARS include infection, hypotension, severe coagulopathy, bleeding, respiratory failure, cardiac failure, acute pancreatitis, severe thrombocytopenia, and seizure. Of these, bleeding complications are closely linked to mortality among liver failure patients [10], and even if donors are obtained, severe coagulopathy, such as disseminated intravascular coagulation (DIC) can impose a major constraint on LT surgery.

Since MARS has been employed in our hospital, bleeding complications have occurred in many patients during or after MARS, even though coagulation-preventing agents, such as heparin or nafamostat, are not administered. One patient died of bleeding complications, and many others could not proceed with additional MARS sessions. However, other patients showed no bleeding-related complications. Therefore, we sought to determine which patients are more likely to experience bleeding complications and any predictive factors. The purpose of this study was to investigate the association between MARS and bleeding complications, and to identify preMARS factors associated with bleeding complications.

\section{MATERIALS AND METHODS}

The Institutional Review Board of Jeonbuk National University Hospital approved this study (IRB No. CUH 2020-03-064). As the study is a retrospective observational one, the need for patient consent was waived.

\section{Data Collection}

Data from all patients receiving MARS in the intensive care

\section{KEY MESSAGES}

- Molecular adsorbent recirculating system (MARS) is a liver support system that can be useful, but bleeding complication needs to be considered.

- MARS was found to cause significant coagulopathy, resulting in increased bleeding complication.

- Low hemoglobin, low factor V, and prolonged international normalized ratio of prothrombin time in the blood test performed before MARS may have a high likelihood of bleeding complication.

unit (ICU) between December 2016 and February 2020 were analyzed retrospectively, which included 17 patients undergoing a total of 41 MARS sessions. Laboratory data, hospitalization records, progress records, ICU record sheets, nursing records, transfusion records, and medication history were reviewed. The following data were collected: patients' demographics; predisposing factors; pre-existing liver disease or bleeding; computed tomography abdomen and biopsy findings; Child-Turcotte-Pugh score, Simplified Acute Physiology Score (SAPS) III, Acute Physiology and Chronic Health Evaluation (APACHE) II score, and model for end-stage liver disease (MELD) score; use of continuous renal replacement therapy (CRRT); need for mechanical ventilation and/or vasopressor support during MARS; timing and duration of MARS sessions; transfused blood units; pre-, on-, and post-MARS complete blood cell count, blood chemistry test, blood coagulation test, and coagulation factors; occurrence of LT; current patient status. Most pre- and post-MARS laboratory values were collected shortly before and after MARS, respectively. However, in cases of packed red blood cell (RBC) transfusion during MARS, some post-MARS laboratory values were replaced by on-MARS laboratory values to exclude the effect of transfusion. Coagulation factor values were obtained pre-MARS, 2 hours after the start of MARS, and post-MARS.

\section{MARS Protoco}

The main reason of MARS application in our cases are treatment of liver failure to enable native liver recovery, or as a bridge to LT. MARS treatment start in patients who has suitable liver transplant recipient with rapid deterioration of hepatic metabolic function, bilirubin $\geq 30 \mathrm{mg} / \mathrm{dl}$ and type 1 hepatorenal syndrome combined hepatic encephalopathy despite usual medical therapy and with or without acute or chronic liver 
disease. However, patients with active bleeding, a tendency to severe bleeding such as DIC and severe thrombocytopenia, or uncontrolled sepsis were excluded $[6,11,12]$. In addition, due to its very high cost, a comprehensive judgment was made considering factors such as the patient's age, possibility of transplantation, and the opinions of the patient or guardians. Eight hours, at least more than 6 hours, of MARS sessions were repeated based on the daily assessment of the intensivist and medical and surgical hepatology specialists until the patient's clinical condition improved.

The Baxter Gambro Mars kit was used, and heparin priming was performed. A Becton Dickinson arterial cannula was inserted into the brachial or radial artery, and an Arrow's You-Bend double-lumen hemodialysis (HD) catheter (12 Fr) for MARS and Arrow's multi-lumen central venous catheter (7 French, 3-lumen) were inserted into the right jugular, subclavian or femoral vein. HD catheter insertion was performed by radiologic intervention specialists under ultrasound and fluoroscopy to minimize the risk of bleeding as well as possible risks. No agent for preventing coagulation, such as heparin or nafamostat, was administered. Packed RBC, platelet concentrate (PC), fresh frozen plasma (FFP), fibrinogen, cryoprecipitate were transfused for suitable hemodynamics and preventing spontaneous bleeding. According to our medical ICU transfusion protocol, regardless of bleeding, blood products transfusions were performed with the target of hemoglobin $\geq 7-8 \mathrm{~g} / \mathrm{dl}$, platelet $\geq 30 \times 10^{3} / \mathrm{mm}^{3}-50 \times 10^{3} / \mathrm{mm}^{3}$, prothrombin time and international normalized ratio (PT-INR) $\leq 2$, and fibrinogen $\geq 150 \mathrm{mg} / \mathrm{dl}$.

\section{Data Analysis and Statistics Analysis}

We first sought to determine the effect of MARS by comparing laboratory values before and after MARS. To identify preMARS factors associated with increased bleeding after MARS, we also divided patients into bleeder and non-bleeder groups, according to whether there was visually confirmed or strong suspicion of bleeding after MARS and compared their preMARS laboratory values. The bleeder group included patients with suddenly increased catheter insertion site hematoma or ecchymosis, hemoglobin reduction by $\geq 2 \mathrm{~g} / \mathrm{dl}$, need for $\mathrm{RBC}$ transfusion with two or more packs, or a marked increase in bleeding after MARS. The non-bleeder group consisted of patients not included in the bleeder group (i.e., patients without catheter insertion site hematoma or ecchymosis, hemoglobin reduction $<2 \mathrm{~g} / \mathrm{dl}$ after MARS, need for RBC transfusion with one or fewer packs, and no marked increase in any bleeding after MARS). We also compared the upper and lower 33\% of MARS sessions based on the amount of hemoglobin reduction after MARS, which were designated as bleeding and non-bleeding sessions, respectively. Based on the results, potential factors associated with hemoglobin change rate were analyzed by multiple linear regression analysis.

Patient demographics and baseline values were compared using independent t-tests or chi-square test, as appropriate. Kolmogorov-Smirnov and Shapiro-Wilk tests were used to determine whether data were normally distributed, after which groups were compared using independent t-tests or Mann-Whitney nonparametric tests. Changes over time in factors were tested using repeated measures analysis of variance. All statistical analysis was performed using IBM SPSS statistics ver. 26 (IBM Corp., Armonk, NY, USA).

\section{RESULTS}

We examined data from 17 patients undergoing a total of 41 MARS sessions (Tables 1 and 2). The average age of patients was 50 years. Twelve were men, and five were women. Five patients had acute liver failure (ALF; 2 hepatitis A virus, 1 alcoholic, 1 pregnancy-induced, 1 toxic hepatitis), and 12 patients had acute-on-chronic liver failure (AoCLF; 9 alcoholic, 3 hepatitis B virus-related liver cirrhosis). MARS was administered one to six times per patient. At the time of MARS application, 13 of 17 patients were receiving either CRRT or HD, and one started CRRT after MARS was terminated. Ten of the 17 patients died, seven were on an outpatient follow-up. Seven patients underwent LT, one of them died 3 days after surgery and the other died 5 months after surgery. Ten patients did not received LT and two of them recovered spontaneously. Thus, two out of five patients with ALF died, two had native liver recovery, and one survived after LT. On the other hand, eight out of 12 patients with AoCLF died and four survived after LT. Forty MARS sessions were uninterrupted, but one MARS session was stopped after 1 hour due to malfunction of the blood leak detector. The average duration of MARS sessions was 8 hours and 18 minutes.

Eight of the 15 patients presented clinical bleeding complications during or after MARS and were classified into the bleeder group. Seven of these eight patients had catheter bleeding, and three had worsening ecchymosis. In two patient, hemoglobin decreased by $<2 \mathrm{~g} / \mathrm{dl}$, and in two patients, more than two packs of RBCs were transfused. One patient exhibited subdural hematoma 3 days after MARS and eventually died. 
Table 1. Patient's data

\begin{tabular}{|c|c|c|c|c|c|c|c|}
\hline No. & $\begin{array}{l}\text { Gender/ } \\
\text { age (yr) }\end{array}$ & $\begin{array}{l}\text { Predisposing } \\
\text { factor }\end{array}$ & Reason for MARS & $\begin{array}{l}\text { No. of } \\
\text { MARS }\end{array}$ & $\begin{array}{l}\text { New or increased bleeding sites } \\
\text { at the time of MARS }\end{array}$ & LT & Final outcome \\
\hline 1 & $\mathrm{M} / 42$ & HAV & $\begin{array}{l}\text { ALF complicated by HEP and renal } \\
\text { dysfunction }\end{array}$ & 3 & No & Yes & Expire after 3 days of LT \\
\hline 2 & $\mathrm{M} / 47$ & Herb & $\begin{array}{l}\text { ALF complicated by HEP and renal } \\
\text { dysfunction }\end{array}$ & 3 & No & Yes & Outpatient FU \\
\hline 3 & $\mathrm{M} / 62$ & Alcohol & $\begin{array}{l}\text { AoCLF complicated by progressive jaundice } \\
\text { and renal dysfunction }\end{array}$ & 3 & Catheter insertion site hematoma & No & Expire \\
\hline 4 & $M / 64$ & Alcohol & $\begin{array}{l}\text { AoCLF complicated by progressive jaundice } \\
\text { and renal dysfunction }\end{array}$ & 2 & No & No & Expire \\
\hline 5 & $F / 28$ & Pregnancy & ALF complicated by renal dysfunction & 1 & $\begin{array}{l}\text { Vaginal bleeding, catheter } \\
\text { insertion site hematoma }\end{array}$ & No & Outpatient FU \\
\hline 6 & $M / 42$ & $\begin{array}{c}\text { Alcohol, GI } \\
\text { bleeding }\end{array}$ & $\begin{array}{l}\text { AoCLF complicated by HEP and renal } \\
\text { dysfunction }\end{array}$ & 1 & $\begin{array}{l}\text { GI bleeding, catheter insertion } \\
\text { site hematoma }\end{array}$ & No & Expire \\
\hline 7 & $\mathrm{~F} / 51$ & HBV & $\begin{array}{l}\text { AoCLF complicated by progressive jaundice } \\
\text { and renal dysfunction }\end{array}$ & 2 & Catheter insertion site hematoma & Yes & Outpatient FU, HD \\
\hline 8 & $M / 62$ & Drug, HBV & $\begin{array}{l}\text { AoCLF complicated by progressive jaundice } \\
\text { and renal dysfunction }\end{array}$ & 2 & No & Yes & Outpatient FU, HD \\
\hline 9 & $\mathrm{~F} / 35$ & HAV & ALF complicated by HEP & 2 & No & No & Outpatient FU \\
\hline 10 & $\mathrm{~F} / 62$ & Alcohol & AoCLF complicated by progressive jaundice & 2 & Brain subdural hemorrhage & No & Expire \\
\hline 11 & $M / 48$ & Alcohol & AoCLF complicated by progressive jaundice & 1 & $\begin{array}{l}\text { Oral bleeding, catheter insertion } \\
\text { site hematoma }\end{array}$ & Yes & Outpatient FU \\
\hline 12 & $M / 59$ & HBV & $\begin{array}{l}\text { AoCLF complicated by progressive jaundice } \\
\text { and renal dysfunction }\end{array}$ & 6 & No & Yes & Outpatient FU, HD \\
\hline 13 & $M / 66$ & Alcohol & AoCLF complicated by progressive jaundice & 2 & Catheter insertion site hematoma & No & Expire \\
\hline 14 & $F / 26$ & Alcohol & AoCLF complicated by progressive jaundice & 3 & No & Yes & $\begin{array}{l}\text { Expire after } 5 \text { months of } \\
\text { LT }\end{array}$ \\
\hline 15 & $\mathrm{M} / 35$ & $\begin{array}{l}\text { Alcohol, Gl } \\
\text { bleeding }\end{array}$ & $\begin{array}{l}\text { AoCLF complicated by HEP, progressive } \\
\text { jaundice, and renal dysfunction }\end{array}$ & 3 & No & No & Expire \\
\hline 16 & $\mathrm{M} / 42$ & HBV & $\begin{array}{l}\text { AoCLF complicated by HEP, progressive } \\
\text { jaundice }\end{array}$ & 2 & No & No & Expire \\
\hline 17 & $\mathrm{M} / 49$ & Alcohol, herb & ALF complicated by HEP, progressive jaundice & 3 & Catheter insertion site hematoma & No & Expire \\
\hline
\end{tabular}

MARS: molecular adsorbent recirculating system; LT: liver transplantation; HAV: hepatitis A virus; ALF: acute liver failure; HEP: hepatic encephalopathy; FU: follow-up; AoCLF: acute-on-chronic liver failure; GI: gastro-intestinal; HBV: hepatitis B virus; HD: hemodialysis.

In addition, various bleeding events including oral bleeding, bloody sputum, hematuria, melena, or vaginal bleeding occurred or worsened in one or more patients. In patients in the bleeder group, hemoglobin decreased by $1.21 \mathrm{~g} / \mathrm{dl}$ after MARS, and they received a daily average of 2.18 packs of RBCs, 7.20 packs of PC, 3.82 packs of FFP, and 5.02 packs of cryoprecipitate during the period receiving MARS (from the beginning of the initial MARS to the day following the end of the last MARS). The remaining nine out of 15 patients were classified into the non-bleeder group. Six patients exhibited little bleeding, and three had ecchymosis and catheter bleeding, but did not show much change after MARS. Hemoglobin before MARS decreased by $0.40 \mathrm{~g} / \mathrm{dl}$ after MARS in the non-bleeder group. During the same period, patients in the non-bleeder group received a daily average of 0.54 packs of RBCs, 2.73 packs of PC, 2.77 packs of FFP, and 2.95 packs of cryoprecipitate.
The amount of change in laboratory values before and after MARS was compared across all patients (Table 3). We found significant changes before and after MARS in hemoglobin, platelet, total bilirubin, direct bilirubin, creatinine, PT-INR, activated partial thromboplastin time (aPTT), fibrinogen, fibrinogen degradation product (FDP), and D-dimer. Total bilirubin and direct bilirubin decreased from 33.0 and $19.5 \mathrm{mg} /$ dl to 28.8 and $16.1 \mathrm{mg} / \mathrm{dl}$ though MARS $(\mathrm{P}<0.001$ and $\mathrm{P}<0.001$, respectively). Creatinine decreased by $0.4 \mathrm{mg} / \mathrm{dl}$ from $1.8 \mathrm{mg} /$ $\mathrm{dl}$ to $1.4 \mathrm{mg} / \mathrm{dl}(\mathrm{P}<0.001)$. Changes in coagulation profiles after MARS were indicative of a negative coagulation effect. That is, PT-INR, aPTT, FDP, and D-dimer significantly increased, and platelet and fibrinogen significantly decreased after MARS. PT-INR and aPTT was 2.4 INR and 48.1 seconds before MARS but increased to 4.6 INR and 77.6 seconds after MARS ( $\mathrm{P}=0.022$ and $\mathrm{P}=0.017$, respectively). Fibrinogen de- 
Table 2. Patient's baseline values before molecular adsorbent recirculating system

\begin{tabular}{|c|c|c|c|c|}
\hline Variable & All patients $(n=17)$ & Bleeder $(n=8)$ & Non-bleeder $(n=9)$ & P-value \\
\hline Male & $12(70.6)$ & $5(62.5)$ & $7(77.8)$ & 0.490 \\
\hline Age (yr) & $50.0 \pm 13.9$ & $51.3 \pm 13.5$ & $46.3 \pm 14.2$ & 0.673 \\
\hline Underlying liver cirrhosis & $12(70.6)$ & $6(75.0)$ & $6(66.7)$ & 0.707 \\
\hline End stage renal disease & 0 & 0 & 0 & 1.000 \\
\hline \multicolumn{5}{|l|}{ Disease severity } \\
\hline CTP score & $10.9 \pm 1.4$ & $10.8 \pm 1.4$ & $11.0 \pm 1.6$ & 0.888 \\
\hline MELD score & $38.5 \pm 6.8$ & $39.9 \pm 5.7$ & $37.3 \pm 7.9$ & 0.481 \\
\hline SAPS III score & $62.3 \pm 10.0$ & $63.0 \pm 10.7$ & $61.7 \pm 10.0$ & 1.000 \\
\hline APACHE II score & $16.1 \pm 6.9$ & $17.5 \pm 7.3$ & $14.9 \pm 6.6$ & 0.481 \\
\hline \multicolumn{5}{|l|}{ Support modality } \\
\hline CRRT & $13(76.5)$ & 7 (87.5) & $6(66.7)$ & 0.312 \\
\hline Mechanical ventilation & $4(23.5)$ & $3(37.5)$ & $1(11.1)$ & 0.079 \\
\hline Vasopressor & $6(35.3)$ & $4(50.0)$ & $2(22.2)$ & 0.079 \\
\hline \multicolumn{5}{|l|}{ Laboratory parameter } \\
\hline Hemoglobin (g/dl) & $9.7 \pm 2.1$ & $9.1 \pm 2.4$ & $10.3 \pm 1.6$ & 0.015 \\
\hline Platelet $\left(\times 10^{3} / \mathrm{mm}^{3}\right)$ & $77.4 \pm 46.2$ & $84.6 \pm 55.6$ & $70.9 \pm 38.2$ & 0.606 \\
\hline AST (IU/L) & $673 \pm 1,360$ & $352 \pm 635$ & $958 \pm 1,776$ & 0.200 \\
\hline ALT (IU/L) & $600 \pm 1,045$ & $359 \pm 678$ & $814 \pm 1,293$ & 0.200 \\
\hline Total bilirubin (mg/dl) & $30.9 \pm 12.7$ & $30.2 \pm 11.7$ & $31.5 \pm 14.3$ & 0.743 \\
\hline Direct bilirubin (mg/dl) & $18.8 \pm 8.9$ & $15.4 \pm 5.9$ & $21.7 \pm 10.4$ & 0.139 \\
\hline Albumin (g/dl) & $3.2 \pm 0.3$ & $3.3 \pm 0.3$ & $3.2 \pm 0.3$ & 0.236 \\
\hline Creatinine (mg/dl) & $1.9 \pm 1.1$ & $1.7 \pm 0.4$ & $2.1 \pm 1.5$ & 0.673 \\
\hline Ammonia $(\mu \mathrm{mol} / \mathrm{L})$ & $89.7 \pm 46.9$ & $91.5 \pm 61.8$ & $88.2 \pm 32.6$ & 0.541 \\
\hline LD (IU/I) & $1,491 \pm 1,692$ & $1,316 \pm 1,348$ & $1,647 \pm 2,019$ & 0.673 \\
\hline PT-INR & $2.5 \pm 0.8$ & $2.7 \pm 1.0$ & $2.3 \pm 0.4$ & 0.606 \\
\hline aPTा (sec) & $48.0 \pm 10.9$ & $46.1 \pm 7.4$ & $49.8 \pm 13.6$ & 0.963 \\
\hline Fibrinogen (mg/dl) & $151.7 \pm 51.6$ & $133.9 \pm 56.4$ & $169.5 \pm 42.4$ & 0.161 \\
\hline $\mathrm{FDP}(\mathrm{mg} / \mathrm{ml})$ & $62.0 \pm 58.4$ & $69.4 \pm 55.7$ & $53.5 \pm 64.6$ & 0.613 \\
\hline Anti-thrombin III (\%) & $28.0 \pm 14.5$ & $25.6 \pm 13.7$ & $30.4 \pm 15.9$ & 0.645 \\
\hline D-dimer (mg/L FEU) & $15.3 \pm 15.7$ & $14.9 \pm 13.2$ & $15.9 \pm 19.3$ & 0.955 \\
\hline Factor II (\%) & $31.5 \pm 7.9$ & $31.3 \pm 6.9$ & $31.7 \pm 9.1$ & 1.000 \\
\hline Factor V (\%) & $27.4 \pm 16.9$ & $16.0 \pm 7.7$ & $35.0 \pm 17.4$ & 0.067 \\
\hline Factor VII (\%) & $19.1 \pm 7.8$ & $17.5 \pm 9.8$ & $20.2 \pm 6.9$ & 0.914 \\
\hline Factor X (\%) & $39.8 \pm 15.4$ & $35.5 \pm 11.1$ & $42.7 \pm 18.2$ & 1.000 \\
\hline Procalcitonin (ng/ml) & $2.0 \pm 1.9$ & $1.3 \pm 0.8$ & $2.6 \pm 2.4$ & 0.321 \\
\hline Lactate (mmol/L) & $3.2 \pm 2.0$ & $3.3 \pm 2.5$ & $3.0 \pm 1.6$ & 0.673 \\
\hline
\end{tabular}

Values are presented as number (\%) or mean \pm standard deviation.

CTP: Child-Turcotte-Pugh; MELD: model for end-stage liver disease; SAPS: Simplified Acute Physiology Score; APACHE: Acute Physiology and Chronic Health Evaluation; CRRT: continuous renal replacement therapy; AST: aspartate transaminase; ALT: alanine aminotransferase; LD: lactate dehydrogenase; PT-INR: international normalized ratio of prothrombin time; aPTT: activated partial thromboplastin time; FDP: fibrinogen degradation product; FEU: fibrinogen equivalent unit.

creased from $155.4 \mathrm{mg} / \mathrm{dl}$ to $98.1 \mathrm{mg} / \mathrm{dl}(\mathrm{P}=0.021)$, and platelet also decreased from $78.8 \times 10^{3} / \mathrm{mm}^{3}$ to $63.5 \times 10^{3} / \mathrm{mm}^{3}(\mathrm{P}=0.002)$. These resulted in a decrease in hemoglobin from $9.4 \mathrm{~g} / \mathrm{dl}$ to 8.8 $\mathrm{g} / \mathrm{dl}(\mathrm{P}<0.001)$. In addition, Figure 1 shows changes in coagu- lation factors measured before MARS, 2 hours after the start of MARS, and after MARS finish. There were no significant differences changes before and after MARS in other chemistry profiles, or inflammatory markers. 
Table 3. Changes in values before and after MARS $(n=41)$

\begin{tabular}{|c|c|c|c|}
\hline Variable & Before MARS & After MARS & P-value \\
\hline \multicolumn{4}{|l|}{ Complete blood count } \\
\hline Hemoglobin (g/dl) & $9.4 \pm 1.7$ & $8.8 \pm 1.8$ & $<0.001$ \\
\hline Platelet $\left(\times 10^{3} / \mathrm{mm}^{3}\right)$ & $78.8 \pm 35.2$ & $63.5 \pm 35.1$ & 0.002 \\
\hline \multicolumn{4}{|l|}{ Chemistry profile } \\
\hline AST (IU/L) & $444 \pm 924$ & $389 \pm 654$ & 0.586 \\
\hline ALT (IU/L) & $470 \pm 836$ & $422 \pm 713$ & 0.126 \\
\hline Total bilirubin (mg/dl) & $33.0 \pm 11.6$ & $28.8 \pm 9.0$ & $<0.001$ \\
\hline Direct bilirubin (mg/dl) & $19.5 \pm 7.7$ & $16.1 \pm 5.7$ & $<0.001$ \\
\hline Albumin (g/dl) & $3.3 \pm 0.4$ & $3.4 \pm 0.5$ & 0.119 \\
\hline Creatinine (mg/dl) & $1.8 \pm 1.0$ & $1.4 \pm 0.7$ & $<0.001$ \\
\hline Ammonia ( $\mu \mathrm{mol} / \mathrm{L})$ & $92.2 \pm 52.5$ & $87.6 \pm 45.7$ & 0.133 \\
\hline LD (IU/L) & $1,320 \pm 1,157$ & $1,266 \pm 787$ & 0.728 \\
\hline \multicolumn{4}{|l|}{ Coagulation profile } \\
\hline PT-INR & $2.4 \pm 0.8$ & $4.6 \pm 6.0$ & 0.022 \\
\hline aPTा (sec) & $48.1 \pm 14.2$ & $77.6 \pm 76.4$ & 0.017 \\
\hline Fibrinogen (mg/dl) & $155.4 \pm 28.1$ & $98.1 \pm 64.1$ & $<0.001$ \\
\hline FDP (mg/ml) & $107 \pm 107$ & $189 \pm 121$ & $<0.001$ \\
\hline Anti-thrombin III (\%) & $30.6 \pm 13.6$ & $34.4 \pm 13.2$ & 0.141 \\
\hline D-dimer (mg/L FEU) & $34.2 \pm 44.8$ & $66.6 \pm 53.6$ & $<0.001$ \\
\hline \multicolumn{4}{|l|}{ Coagulation factor } \\
\hline Factor II (\%) & $34.7 \pm 9.3$ & $32.0 \pm 10.6$ & 0.014 \\
\hline Factor V (\%) & $30.1 \pm 17.6$ & $21.9 \pm 17.1$ & $<0.001$ \\
\hline Factor VII (\%) & $21.2 \pm 9.8$ & $19.7 \pm 9.3$ & 0.008 \\
\hline Factor X (\%) & $44.4 \pm 16.0$ & $41.4 \pm 16.3$ & 0.003 \\
\hline
\end{tabular}

Values are presented as mean \pm standard deviation.

MARS: molecular adsorbent recirculating system; AST: aspartate transaminase; ALT: alanine aminotransferase; LD: lactate dehydrogenase; PT-INR: international normalized ratio of prothrombin time; aPTT: activated partial thromboplastin time; FDP: fibrinogen degradation product; FEU: fibrinogen equivalent unit.

Table 2 presents pre-MARS values for patients in the bleeder and non-bleeder groups. Pre-MARS hemoglobin was significantly lower in the bleeder group $(9.1 \mathrm{~g} / \mathrm{dl})$ than in the non-bleeder group (10.3 g/dl; $\mathrm{P}=0.015)$. Apart from hemoglobin, however, there were no significant differences between patient groups.

Table 4 shows a comparison of the upper and lower 33\% of MARS sessions based on hemoglobin reduction rate. After MARS, hemoglobin decreased by $1.49 \mathrm{~g} / \mathrm{dl}$ on average in the upper $33 \%$ of sessions (i.e., bleeding sessions) and increased by $0.15 \mathrm{~g} / \mathrm{dl}$ in the lower $33 \%$ of sessions (i.e., non-bleeding sessions). Pre-MARS PT-INR was significantly higher for bleeding sessions (2.7 INR) than for non-bleeding sessions (2.0 INR; $\mathrm{P}=0.038$ ). Also, pre-MARS factor $\mathrm{V}$ was significantly lower

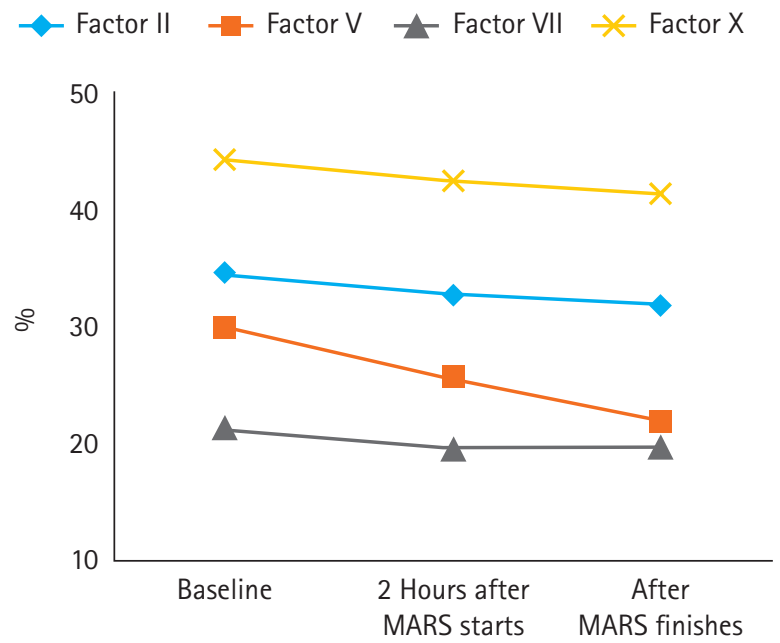

Figure 1. Sequential measurements of coagulation factors $(n=27)$. All factors (factor II, V, VII, and X) decreased significantly over time ( $P=0.012, P<0.001, P=0.001$, and $P=0.002$, respectively). Note that factor $V$ sharply decreased from $30.1 \%$ before molecular adsorbent recirculating system (MARS) to $25.8 \%$ and $21.9 \%$, respectively, 2 hours after MARS started and after MARS finished.

for bleeding sessions (24.8\%) than for non-bleeding sessions (45.6\%; $\mathrm{P}=0.023)$. Other values did not significantly differ between groups.

Multiple linear regression analysis was performed to verify the effect of pre-MARS factors potentially associated with hemoglobin change rate through MARS (Table 5). The results of verifying the significance of the regression coefficient show that pre-MARS hemoglobin, PT-INR, and factor V have a significant effect on the hemoglobin change rate, whereas pre-MARS platelet, total bilirubin, albumin, fibrinogen, and anti-thrombin do not. That is, the $\mathrm{B}$ value of the pre-MARS PT-INR was -5.227 ( $\mathrm{P}=0.036)$, and as the pre-MARS PT-INR increased by 1 INR, the post-MARS hemoglobin decreased by $5.227 \%$. The decrease of $1 \mathrm{~g} / \mathrm{dl}$ hemoglobin and $1 \%$ factor $\mathrm{V}$ in pre-MARS can also be predicted to decrease post-MARS hemoglobin by $2.443 \%$ and $0.281 \%$, respectively.

\section{DISCUSSION}

Our results show that MARS acts as a liver support system by reducing total bilirubin, direct bilirubin, and creatinine. However, MARS may increase the risk of bleeding by increasing PT and aPTT and reducing platelet and fibrinogen. As a result, hemoglobin levels decreased by $0.6 \mathrm{~g} / \mathrm{dl}$ on average after MARS compared to before MARS. When comparing MARS sessions between two groups according to bleeding and hemoglobin 
Table 4. Pre-MARS values for bleeding and non-bleeding sessions based on rate of hemoglobin decrease during MARS

\begin{tabular}{|c|c|c|c|}
\hline Variable & $\begin{array}{c}\text { Bleeding } \\
\text { sessions }(n=14)\end{array}$ & $\begin{array}{l}\text { Non-bleeding } \\
\text { sessions }(n=13)\end{array}$ & P-value \\
\hline \multicolumn{4}{|l|}{ Support modality } \\
\hline CRRT & $11(78.6)$ & $9(69.2)$ & 0.685 \\
\hline Mechanical ventilation & $4(28.6)$ & $1(7.7)$ & 0.375 \\
\hline Vasopressor & $4(28.6)$ & $1(7.7)$ & 0.375 \\
\hline \multicolumn{4}{|l|}{ Complete blood count } \\
\hline Hemoglobin (g/dl) & $9.4 \pm 2.2$ & $10.1 \pm 1.5$ & 0.054 \\
\hline Platelet $\left(\times 10^{3} / \mathrm{mm}^{3}\right)$ & $87.2 \pm 41.3$ & $89.7 \pm 30.4$ & 0.616 \\
\hline \multicolumn{4}{|l|}{ Laboratory parameter } \\
\hline AST (IU/L) & $347 \pm 499$ & $857 \pm 1506$ & 0.519 \\
\hline ALT (IU/L) & $350 \pm 599$ & $986 \pm 1213$ & 0.239 \\
\hline Total bilirubin (mg/dl) & $34.1 \pm 11.1$ & $25.1 \pm 13.3$ & 0.066 \\
\hline Direct bilirubin (mg/dl) & $19.6 \pm 7.7$ & $16.7 \pm 9.1$ & 0.379 \\
\hline Albumin (g/dl) & $3.3 \pm 0.4$ & $3.4 \pm 0.4$ & 0.820 \\
\hline Creatinine (mg/dl) & $1.8 \pm 0.9$ & $1.5 \pm 0.8$ & 0.759 \\
\hline Ammonia $(\mu \mathrm{mol} / \mathrm{L})$ & $95.5 \pm 72.0$ & $96.0 \pm 40.9$ & 0.350 \\
\hline LD (IU/L) & $1,265 \pm 1,088$ & $1,534 \pm 1,690$ & 0.943 \\
\hline \multicolumn{4}{|l|}{ Coagulation profile } \\
\hline PT-INR & $2.7 \pm 1.1$ & $2.0 \pm 0.3$ & 0.038 \\
\hline aPTा (sec) & $46.4 \pm 5.8$ & $45.5 \pm 13.5$ & 0.141 \\
\hline Fibrinogen (mg/dl) & $163 \pm 84$ & $159 \pm 43$ & 0.859 \\
\hline $\mathrm{FDP}(\mathrm{mg} / \mathrm{ml})$ & $106.9 \pm 106.8$ & $98.4 \pm 98.7$ & 0.810 \\
\hline Anti-thrombin III (\%) & $26.6 \pm 12.4$ & $26.4 \pm 7.7$ & 0.955 \\
\hline D-dimer (mg/L FEU) & $32.1 \pm 45.6$ & $32.3 \pm 39.5$ & 0.689 \\
\hline \multicolumn{4}{|l|}{ Coagulation factor } \\
\hline Factor II (\%) & $33.5 \pm 8.8$ & $39.6 \pm 10.8$ & 0.270 \\
\hline Factor V (\%) & $24.8 \pm 16.8$ & $45.6 \pm 21.2$ & 0.023 \\
\hline Factor VII (\%) & $19.3 \pm 9.2$ & $25.0 \pm 8.3$ & 0.222 \\
\hline Factor X (\%) & $41.5 \pm 15.2$ & $52.3 \pm 18.9$ & 0.212 \\
\hline
\end{tabular}

Values are presented as number (\%) or mean \pm standard deviation. MARS: molecular adsorbent recirculating system; CRRT: continuous renal replacement therapy; AST: aspartate transaminase; ALT: alanine aminotransferase; LD: lactate dehydrogenase; PT-INR: international normalized ratio of prothrombin time; aPTT: activated partial thromboplastin time; FDP: fibrinogen degradation product; FEU: fibrinogen equivalent unit.

change, parameters that differed significantly between two groups were pre-MARS hemoglobin, PT-INR, and factor V, and multiple linear regression analysis also indicates that these three factors are independently related to the hemoglobin change rate. This suggests that low hemoglobin, low factor $\mathrm{V}$, and prolonged PT-INR in the blood test performed before MARS may have a high likelihood of bleeding complication.

Lower pre-MARS hemoglobin in the bleeding group suggests that bleeding, including micro-bleeding, may have been
Table 5. Multiple linear regression analysis of pre-MARS factors potentially associated with hemoglobin change rate (\%)

\begin{tabular}{lcccc}
\hline \multirow{2}{*}{ Independent variable } & \multicolumn{2}{c}{$\begin{array}{c}\text { Unstandardized } \\
\text { coefficient }\end{array}$} & $\begin{array}{c}\text { Standardized } \\
\text { coefficient }\end{array}$ & P \\
\cline { 2 - 4 } & $\mathrm{B}$ & $\mathrm{SE}$ & $\beta$ & \\
\hline Hemoglobin $(\mathrm{g} / \mathrm{dl})$ & 2.443 & 0.994 & 0.460 & 0.020 \\
Platelet $\left(\times 10^{3} / \mathrm{mm}^{3}\right)$ & -0.072 & 0.046 & -0.281 & 0.132 \\
Total bilirubin $(\mathrm{mg} / \mathrm{dl})$ & 0.153 & 0.157 & 0.198 & 0.338 \\
Albumin $(\mathrm{g} / \mathrm{dl})$ & 6.657 & 3.948 & 0.280 & 0.101 \\
PT-INR & -5.227 & 2.389 & -0.462 & 0.036 \\
Fibrinogen $(\mathrm{mg} / \mathrm{dl})$ & -0.030 & 0.027 & -0.185 & 0.284 \\
Anti-thrombin III $(\%)$ & -0.132 & 0.102 & -0.199 & 0.206 \\
Factor V $(\%)$ & 0.281 & 0.125 & 0.434 & 0.032 \\
\hline
\end{tabular}

$F=2.582(P=0.027), a_{j} R^{2}=0.240$, Durbin-Watson=2.154.

MARS: molecular adsorbent recirculating system; SE: standard error; PT-INR: international normalized ratio of prothrombin time.

present prior to MARS, unless the patient had underlying anemia, chronic kidney disease, or hematological disease. There were no differences in pre-MARS parameters between bleeder and non-bleeder groups except for hemoglobin, and no patient had underlying chronic kidney disease. Thus, our results suggest that MARS increases bleeding risk when a tendency toward bleeding already exists. Pre-MARS platelet count, PT, and aPTT did not significantly differ between groups. As individual differences in bleeding tendency exist, laboratory values may not fully reflect a patient's bleeding tendency.

The liver is the site where fibrinogen and factors II, V, VII, IX, X, XI, and XII are synthesized. In patients with hepatic insufficiency, levels of these factors are low due to protein dysfunction and poor synthetic function. Defects in $\gamma$-carboxy-glutamic acid residues introduced by vitamin K-dependent carboxylase result in deterioration of the functions of factor II, VII, IX, and X and proteins C and S. By contrast, although factor $\mathrm{V}$ is mainly synthesized in the liver, it is a vitamin K- independent coagulation factor [13]. This may be the basis for the fact that the plasma half-life of factor $\mathrm{V}$ is relatively short, about 12-24 hours, and a decrease in factor $\mathrm{V}$ is the most sensitive indicator of liver failure [14,15]. Many studies also suggest that factor $\mathrm{V}$ levels are related to survival and can serve as a prognostic indicator [16-18]. Interestingly, the gene for factor $V$ related to the family of multi-copper oxidases, and is homologous to coagulation factor VIII. Activated factor $\mathrm{V}$ (factor $\mathrm{Va}$ ) is a major cofactor of the prothrombinase complex. The activated factor $\mathrm{X}$ enzyme requires calcium and factor Va to convert prothrombin to thrombin on the cell surface membrane. Factor Va is degraded by activated protein C, 
one of the principal physiological inhibitors of coagulation. In the presence of thrombomodulin, thrombin acts to decrease clotting by activating protein $\mathrm{C}$; therefore, the concentration and action of protein $\mathrm{C}$ are important determinants in the negative feedback loop through which thrombin limits its own activation. Because this study was a simple retrospective observational study, we did not predict the association of factor $\mathrm{V}$ related-MARS complication and not check the protein $\mathrm{C}$ and von Willebrand factor. There is a limitation in studying additional associations such as protein $\mathrm{C}$ and other factors related to coagulation.

Many studies suggest that MARS reduces bilirubin and creatinine and improves hepatic encephalopathy [3]. However, its side effects such as bleeding complication and survival rate are somewhat controversial. A meta-analysis published in 2012 [5] reported that MARS reduced total bilirubin and clinical symptoms of hepatic encephalopathy, but had no effect on survival. In a meta-analysis published in 2019 [7], there was no difference in the survival of patients receiving standard medical support or MARS, but patients receiving high-intensity therapy with five or more MARS sessions showed better survival than those receiving low-intensity therapy with four or fewer sessions. They also found that the incidence of bleeding complications in the MARS group was $24.1 \%$ compared to $10.2 \%$ of patients receiving standard medical care $(\mathrm{P}=0.007)$. A meta-analysis published in 2020 [19] reports that MARS reduces mortality (relative risk [RR], 0.84; moderate certainty) among patients with ALF or AoCLF and is associated with bleeding-related side effects, such as hypotension (RR, 1.46; low certainty), bleeding (RR, 1.21; moderate certainty), and thrombocytopenia (RR, 1.62; very low certainty). Bleeding complications of MARS can induce an unstable hemodynamic state, increase blood transfusion demand, and impede the procedure [20,21]. Bleeding complications can also promote DIC in patients with liver failure, making medical staff hesitant to proceed with LT [10].

Many recent studies of MARS mention bleeding-associated side effects, such as thrombocytopenia, coagulopathy, hypofibrinogenemia, and anemia, but few studies focus on these outcomes. Among them, several studies using viscoelastic tests such as thromboelastography (TEG) or rotational thromboelastometry (ROTEM) indicate that MARS induces platelet-mediated coagulopathy, both mechanically and immunologically [22,23]. MARS is an extracorporeal circulation system that potentially activates coagulation by putting blood in contact with artificial materials, resulting in the consumption of coagulation factors and platelets. We found that MARS significantly reduced platelet count by $15.3 \times 10^{3} / \mathrm{mm}^{3}$ and also reduced coagulation factors, especially factor V. Moreover, the large-bore venous cannulation ( $12 \mathrm{Fr}$ ) required for MARS may be a factor that causes bleeding in liver failure patients with coagulopathy. In this study, nine out of 17 patients (53\%) had a bleeding complication, and eight out of nine patients (89\%) had muscle or subcutaneous bleeding or hematoma related to catheter insertion. This differs significantly from the study in patients with ALF who received supportive care without MARS. The study found a $10.6 \%$ incidence of bleeding complications, of which $11 \%$ were procedure-related [10]. Double-lumen HD catheter (12Fr) and arterial line catheters were inserted in all patients so that bleeding tendency could be evaluated under the similar conditions. Unlike previous studies, we did not use heparin during MARS treatment and controlled the effects of blood transfusions such as packed RBCs, PC, and FFP.

Our study has several limitations. First, the number of patients was somewhat small, and the data collection period was long. Second, Due to its retrospective design, the time that laboratory testing was performed differed slightly across patients. Moreover, there was an inevitable difference in timing because we had to select two time points between which blood transfusion was not performed. Third, we cannot rule out the subjectivity of the researcher, as we grouped patients primarily based on their visible bleeding. The frequency of bleeding complications after MARS in our study was much higher than that in previous studies, even though heparin was not used. To explain this discrepancy, we reviewed the MARS procedure several times and contacted the MARS kit manufacturer to ensure that there were no changes in the kit or its recommended use instructions. Forth, as a retrospective study, viscoelastic tests such as TEG or ROTEM were not performed during the study period. These were not our routine practice.

In conclusion, MARS could appears to alter coagulation-related factors, such as platelet count, PT, aPTT, fibrinogen, and coagulation factors, and increase the tendency toward bleeding complications particularly in patients with low hemoglobin. Therefore, the progression of coagulopathy should be considered when proceeding with MARS. However, the differences observed among patients were large, and various factors, such as pre-MARS hemoglobin, PT-INR and factor V, appear to contribute to these differences. Further research on this subject is warranted given that the ability to predict bleeding complications, before their onset, would be useful to clinicians when making decisions regarding the use of MARS. 


\section{CONFLICT OF INTEREST}

No potential conflict of interest relevant to this article was reported.

\section{ACKNOWLEDGMENTS}

This paper was presented at the Acute and Critical Care Conference 2020 and was supported by Fund of Biomedical Research Institute, Jeonbuk National University Hospital.

We would like to thank the continuous renal replacement therapy nurse and medical intensive care unit nurses for their assistance with this study.

\section{ORCID}

$\begin{array}{ll}\text { Seon Woo Yoo } & \text { https://orcid.org/0000-0002-1742-7487 } \\ \text { Min-Jong Ki } & \text { https://orcid.org/0000-0001-9959-7908 } \\ \text { Dal Kim } & \text { https://orcid.org/0000-0002-4411-2755 } \\ \text { Seul Ki Kim } & \text { https://orcid.org/0000-0003-2804-2467 } \\ \text { SeungYong Park } & \text { https://orcid.org/0000-0002-3774-4375 } \\ \text { Hyo Jin Han } & \text { https://orcid.org/0000-0003-1601-0724 } \\ \text { Heung Bum Lee } & \text { https://orcid.org/0000-0002-8267-8434 }\end{array}$

\section{AUTHOR CONTRIBUTIONS}

Conceptualization: HBL. Data curation: DK, SKK. Formal analysis: HJH. Methodology: SYP. Project administration: MJK. Visualization: MJK. Writing-original draft: SWY. Writing-review \& editing: MJK, HBL.

\section{REFERENCES}

1. Nanchal R, Subramanian R, Karvellas CJ, Hollenberg SM, Peppard WJ, Singbartl K, et al. Guidelines for the management of adult acute and acute-on-chronic liver failure in the ICU: cardiovascular, endocrine, hematologic, pulmonary and renal considerations: executive summary. Crit Care Med 2020;48:415-9.

2. Lim YS. Acute liver failure in Korea: etiology, prognosis and treatment. Korean J Hepatol 2010;16:5-18.

3. Gerth HU, Pohlen M, Thölking G, Pavenstädt H, Brand M, Hüsing-Kabar A, et al. Molecular adsorbent recirculating system can reduce short-term mortality among patients with acuteon-chronic liver failure-a retrospective analysis. Crit Care Med 2017;45:1616-24.
4. Choi JW, Yoon KT, Park JY, Kim JK, Ahn SH, Paik YH, et al. Usefulness and safety of extracorporeal liver support therapy using MARSR for patients with liver failure: a preliminary report. Korean J Gastroenterol 2009;54:28-35.

5. Vaid A, Chweich H, Balk EM, Jaber BL. Molecular adsorbent recirculating system as artificial support therapy for liver failure: a meta-analysis. ASAIO J 2012;58:51-9.

6. Saliba F. The Molecular Adsorbent Recirculating System (MARS) in the intensive care unit: a rescue therapy for patients with hepatic failure. Crit Care 2006;10:118.

7. Bañares R, Ibáñez-Samaniego L, Torner JM, Pavesi M, Olmedo C, Catalina MV, et al. Meta-analysis of individual patient data of albumin dialysis in acute-on-chronic liver failure: focus on treatment intensity. Therap Adv Gastroenterol 2019;12:1756284819879565.

8. He GL, Feng L, Duan CY, Hu X, Zhou CJ, Cheng Y, et al. Meta-analysis of survival with the molecular adsorbent recirculating system for liver failure. Int J Clin Exp Med 2015;8:17046-54.

9. Khuroo MS, Khuroo MS, Farahat KL. Molecular adsorbent recirculating system for acute and acute-on-chronic liver failure: a meta-analysis. Liver Transpl 2004;10:1099-106.

10. Stravitz RT, Ellerbe C, Durkalski V, Schilsky M, Fontana RJ, Peterseim C, et al. Bleeding complications in acute liver failure. Hepatology 2018;67:1931-42.

11. Boyle M, Kurtovic J, Bihari D, Riordan S, Steiner C. Equipment review: the molecular adsorbents recirculating system (MARS). Crit Care 2004;8:280-6.

12. Jalan R, Sen S, Williams R. Prospects for extracorporeal liver support. Gut 2004;53:890-8.

13. Duga S, Asselta R, Tenchini ML. Coagulation factor V. Int J Biochem Cell Biol 2004;36:1393-9.

14. Izumi S, Langley PG, Wendon J, Ellis AJ, Pernambuco RB, Hughes RD, et al. Coagulation factor V levels as a prognostic indicator in fulminant hepatic failure. Hepatology 1996;23:150711.

15. Bernuau J, Goudeau A, Poynard T, Dubois F, Lesage G, Yvonnet $\mathrm{B}$, et al. Multivariate analysis of prognostic factors in fulminant hepatitis B. Hepatology 1986;6:648-51.

16. Ichai P, Laurent-Bellue A, Saliba F, Moreau D, Besch C, Francoz C, et al. Acute liver failure/injury related to drug reaction with eosinophilia and systemic symptoms: outcomes and prognostic factors. Transplantation 2017;101:1830-7.

17. Zulian MC, Chedid MF, Chedid AD, Grezzana Filho TJ, Leipnitz I, de Araujo A, et al. Low serum factor V level: early predictor of allograft failure and death following liver transplantation. Langenbecks Arch Surg 2015;400:589-97. 
18. Elinav E, Ben-Dov I, Hai-Am E, Ackerman Z, Ofran Y. The predictive value of admission and follow up factor $\mathrm{V}$ and VII levels in patients with acute hepatitis and coagulopathy. J Hepatol 2005;42:82-6.

19. Alshamsi F, Alshammari K, Belley-Cote E, Dionne J, Albrahim T, Albudoor B, et al. Extracorporeal liver support in patients with liver failure: a systematic review and meta-analysis of randomized trials. Intensive Care Med 2020;46:1-16.

20. Kim Y, Kim CK, Jung S, Ko SB. Brain oxygen monitoring via jugular venous oxygen saturation in a patient with fulminant hepatic failure. Korean J Crit Care Med 2016;31:251-5.
21. Ha SJ, Hwang YJ, Lim DG. Hemodynamic changes during isolated liver hemoperfusion of hepatoma. Korean J Crit Care Med 2004;19:115-20.

22. Faybik P, Bacher A, Kozek-Langenecker SA, Steltzer H, Krenn CG, Unger S, et al. Molecular adsorbent recirculating system and hemostasis in patients at high risk of bleeding: an observational study. Crit Care 2006;10:R24.

23. Doria C, Mandalà L, Smith JD, Caruana G, Scott VL, Gruttadauria S, et al. Thromboelastography used to assess coagulation during treatment with molecular adsorbent recirculating system. Clin Transplant 2004;18:365-71. 